
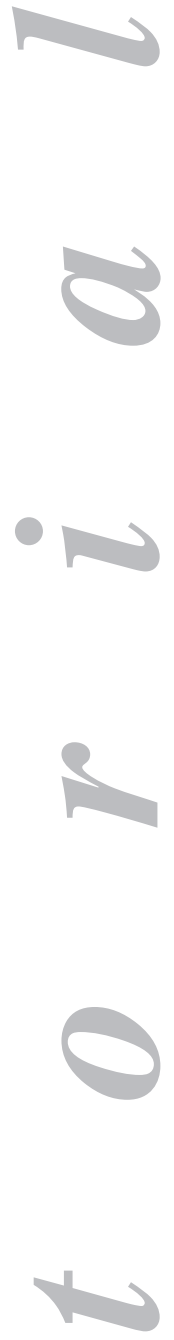

(
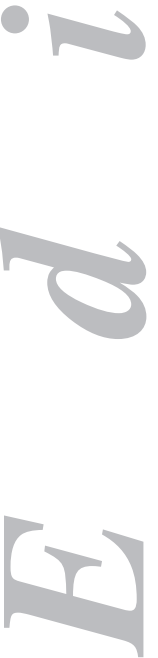

\section{Granulocitoaféresis: viejos procedimientos, nuevas soluciones}

La enfermedad inflamatoria intestinal (EII) es un proceso inflamatorio crónico que afecta a millones de individuos en el mundo, la mayoría de ellos diagnosticados antes de los 30 años y que precisarán un tratamiento a largo plazo. Ante esta situación, es necesaria la búsqueda de nuevos tratamientos que eviten la cirugía, provoquen escasos efectos secundarios y originen el menor impacto posible en la calidad de vida del individuo. En las dos últimas décadas se han producido grandes avances en el conocimiento de la inmunopatogénesis de la EII y se han desarrollado terapias dirigidas a bloquear selectivamente la respuesta inmune en puntos específicos (citoquinas, moléculas de adhesión, etc.). Estos avances han ampliado de forma importante el arsenal terapéutico para el control de la EII. Sin embargo, la situación actual dista mucho de ser ideal ya que, a pesar de disponer de inmunosupresores no selectivos (5-ASA y esteroides), inmunomoduladores (azatiprina, 6-mercaptopurina y metotrexato) y terapias biológicas (anti-TNF $\alpha$, anti-integrinas, etc.), alrededor de un $60 \%$ de los pacientes con enfermedad de Crohn (EC) y aproximadamente un $35 \%$ de los pacientes con colitis ulcerosa $(\mathrm{CU})$ requieren tratamiento quirúrgico $(1,2)$. Tampoco pueden ignorarse los efectos secundarios de los tratamientos actuales.

En los últimos años, se ha introducido una variante de las técnicas de aféresis en el tratamiento de la EII. Se trata de la aféresis leucocitaria que consiste en la extracción de los leucocitos del torrente circulatorio mediante el paso de la sangre a través de un sistema extracorpóreo, modulando así la repuesta inmune. En el momento actual en Japón y en Europa hay dos sistemas comercializados de citoaféresis para el tratamiento de la EII y otras enfermedades autoinmunes: el Adacolum ${ }^{\circledR}$ (Japan Immunoresearch Laboratories, Takasaki, Japón) y el Cellsorba ${ }^{\circledR}$. El primero realiza una aféresis selectiva de granulocitos/monocitos y de una mínima fracción de linfocitos. Esta granulocitoaféresis (GMCAP) se obtiene por el paso de la sangre a través de una columna que contiene gránulos de diacetato de celulosa $(3,4)$. El Cellsorba (Asahi Kasei Medical Co. Ltd, Tokio, Japón) consiste en un filtro formado por una membrana de poliéster que provoca una leucocitoaféresis (LCAP), ya que atrapa de forma no selectiva el $90 \%$ de los leucocitos (100 de granulocitos, 60 de linfocitos y $35 \%$ de las plaquetas) (5). En nuestro país el de uso más extendido es el primero.

Actualmente, aún no se conoce de forma exacta el mecanismo de acción de la granulocitoaféresis. Sabemos que los granulocitos y los monocitos/macrófagos se encuentran elevados y activados en sangre periférica y en la mucosa intestinal durante los brotes de EII, que su número se correlaciona con la actividad y con la gravedad de la enfermedad (6) y que desempeñan un papel clave en la inmunopatogénesis de la EII, ya que producen elevadas cantidades de citoquinas inflamatorias reponsables de la lesión intestinal (7). Estas células constituyen la primera diana de la GMCAP. Sin embargo, su disminución difícilmente explicaría la mejoría a largo 
plazo de los pacientes tras suspender el tratamiento (8) por lo que deben intervenir otros factores. En este sentido, Suzuki y cols., tras la GMCAP, encuentran un importante incremento en el plasma de los niveles de los receptores solubles I y II del TNF $\alpha$ que posiblemente actúen neutralizando la función del TNF $\alpha$ (9). Además, los leucocitos en sangre periférica tras la GMCAP producen menos citoquinas proinflamatorias (TNFa, IL-1, IL-6 e IL-8) (10-12). Conocemos también que la GMCAP origina una disminución en los leucocitos de la expresión de L-selectina y del receptor CXCR3 y un incremento de la integrina Mac-1 y que, por lo tanto, suprimiría la extravasación leucocitaria a nivel de la mucosa intestinal $(11,13)$. Respecto a esta última, los estudios existentes han mostrado una disminución de los niveles de IFN- $\gamma$ producidos por las células $\mathrm{T}$ a nivel intestinal y que los niveles bajos de este predicen tanto la respuesta como el mantenimiento de la remisión a largo plazo (14). Los pacientes que alcanzan la remisión clínica tras la GMCAP presentan bajas concentraciones de IL-1 $\beta$, IL-1ra, IL-6, IL-8 Y TNF- $\alpha$ en la mucosa intestinal, mientras que la relación IL-1ra/IL-1 $\beta$ se encuentra muy incrementada (7).

También se ha observado que la granulocitoaféresis con Adacolum incrementa el número de linfocitos en sangre periférica y parece que este aumento se produce a expensas de los linfocitos T CD4+. De estos linfocitos, el subgrupo de linfocitos T CD4+CD25+ son células T reguladoras que suprimen la inflamación intestinal mediante mecanismos que implican a la IL-10 y al factor transformante del crecimiento $\beta$ (TGF $\beta)(9,11,15,16)$. Recientemente, se ha publicado un trabajo que analiza el papel de las células $\mathrm{T}$ reguladoras para suprimir las respuestas inflamatorias dirigidas hacia autoantígenos y antígenos de las bacterias intestinales endógenas, planteando nuevas estrategias terapéuticas para la EII. Estas incluirían la extracción selectiva por aféresis de linfocitos de sangre periférica seguida de una diferenciación forzada de los mismos al ser expuestos a determinadas citoquinas, compuestos tolerogénicos o a la inclusión en los mismos de un gen regulador. Posteriormente estos serían readministrados al paciente para conseguir una regulación a la baja del proceso inflamatorio (17). En resumen, aunque no se conoce con exactitud el mecanismo de acción de la GMCAP, podemos señalar que se trata de un tratamiento inmunomodulador tras el cual, junto con la disminución del número de granulocitos/monocitos y el aumento en el número de granulocitos inmaduros en sangre periférica, se produce un cambio funcional en estas células con disminución de citoquinas proinflamatorias, aumento de citoquinas antiinflamatorias y disminución de la expresión de las moléculas de adhesión en los granulocitos y, en consecuencia, una menor migración hacia la mucosa intestinal de los mismos.

La granulocitoaféresis se encuentra indicada para el tratamiento de la CU y con menor grado de evidencia en la EC $(7,18)$. En la colitis ulcerosa se ha demostrado que es eficaz para inducir la remisión de pacientes corticodependientes, corticorrefractarios y pacientes naïve de esteroides. Para conocer el alcance de dicho tratamiento es interesante reseñar algunos estudios. En pacientes corticorresistentes con brote grave, Hanai y cols. (19) encontraron tasas de respuesta de hasta el $80 \%$, con un $79 \%$ de pacientes en remisión a largo plazo frente al $64 \%$ de los tratados con ciclosporina (20). Otros estudios han mostrado resultados similares (21-24). En la CU corticodependiente se ha mostrado eficaz, ya que se ha conseguido la retirada de los esteroides o una importante reducción de los mismos en el 60-68\% de los pacientes $(22,25)$. La GMCAP ha resultado ser muy eficaz en la inducción de la remisión en pacientes con $\mathrm{CU}$ que no habían sido tratados previamente con esteroides, con tasas de respuesta en el 85-100\% de los casos $(9,23)$. En la serie de Suzuki los pacientes presentaban una CU moderada-grave y el número de aféresis fue inicialmente de 
2 por semana hasta conseguir la mejoría tras un total de 10 sesiones. Todos los pacientes recibían 5-ASA (9). Resultados similares obtuvieron Yamamoto y cols. en la CU izquierda (23). En una publicación de Bresci y cols. (26) que compara la eficacia de la GMCAP frente a la metil-prednisolona con el fin de inducir la remisión en un brote de CU, se menciona que los pacientes tratados con GMCAP conseguían la remisión en el $70 \%$ de los casos y mejoría en el $20 \%$ frente al $60 \%$ de remisiones y al $10 \%$ de mejoría del grupo tratado con esteroides. Existen trabajos que muestran una remisión al menos durante 18 meses en el 60\% de los pacientes corticorrefractarios (27).

En la EC su eficacia es menos conocida ya que se han realizado pocos estudios y los grupos no son homogéneos en cuanto a localización y patrón de la enfermedad. Las tasas de respuesta obtenidas en los diferentes estudios en la EC corticodependiente y corticorresistente oscilan entre el 52 y el 70\% (28-31). Doménech y cols., en 10 pacientes con EC corticodependiente, logran la remisión tanto clínica como endoscópica en el $70 \%$ de los casos. Sin embargo, únicamente un paciente mantenía la respuesta al año del tratamiento (31).

Este número de la Revista Española de Enfermedades Digestivas incluye un interesante análisis retrospectivo de los resultados obtenidos en 10 pacientes con CU y 8 con EC tratados con la GMCAP. En este estudio, Fernández-Pérez y cols. analizan la eficacia de dicho tratamiento en la inducción de la remisión y en el mantenimiento de la misma en las dos variedades de EII. Todos los pacientes presentaban una enfermedad inflamatoria intestinal corticodependiente o corticorresistente. En los pacientes con CU obtuvieron resultados similares a los referidos en la literatura, mientras que en la EC el porcentaje de pacientes en remisión a la 32 semana era mayor que en la CU (32). Debido, sin embargo, al reducido número de pacientes y al carácter heterogéneo de los mismos es difícil extraer conclusiones definitivas.

Las recomendaciones de GETECCU (Grupo Español para el Estudio de la EC y CU) para la utilización de la granulocitoaféresis han sido publicadas en un documento de consenso (33).

A la vista de los resultados de los estudios realizados, la granulocitoaféresis es una técnica segura con escasos y leves efectos secundarios que aparecen en el 5$27 \%$ de los pacientes. Los más frecuentes son los vértigos, náuseas, cefaleas, enrojecimiento y la fiebre (34). Se ha publicado un caso de alteración transitoria y autolimitada de las pruebas de función hepática que se relacionó con el anticoagulante empleado (mesilato de nafamostat) (23). Dada su buena tolerancia, la mayor parte de los pacientes suelen completar el tratamiento. El principal problema de la aféresis lo constituyen los accesos venosos que en pacientes tratados con esteroides y con múltiples ingresos pueden ser difíciles de obtener, siendo necesario en estos casos invidualizar el acceso a una vía central, con el consiguiente riesgo de complicaciones infecciosas. Debido a su mecanismo de acción, no es previsible que se produzca pérdida de la eficacia o dependencia del mismo.

La GMCAP está contraindicada en los casos de anemia grave, enfermedad cardiaca y renal y en alérgicos a la heparina. Sus escasos efectos secundarios la sitúan como una importante opción terapéutica en el manejo de la CU en pediatría. Se han publicado varios trabajos en este grupo de pacientes, demostrando que la técnica es segura y útil para el control del brote y el descenso de la dosis de esteroides $(35,36)$. También se encuentra contraindicada en mujeres embarazadas. Sin embargo, recientemente se ha publicado un caso en el que se ha mostrado eficaz y segura, sin daños en el feto ni en la evolución de la gestación, controlando un brote grave de CU (37).

Pese a todos estos estudios, aún queda por definir cuál es la pauta terapéutica idónea en cuanto al número y duración de las sesiones de aféresis. Actualmente, el 
tratamiento estándar consiste en una sesión semanal (de una hora de duración) durante 5 semanas. Si bien existen estudios que demuestran que dos sesiones semanales aceleran la remisión del proceso inflamatorio y disminuyen las necesidades de esteroides, esta pauta no implica el aumento del número de pacientes respondedores $(38,39)$. Trabajos posteriores parecen indicar que es más eficaz una pauta de, al menos, 10 sesiones de 90 minutos de duración, pudiendo en las primeras semanas aplicar dos sesiones semanales (33). Todo ello nos conduce a uno de los problemas que ha dificultado la implantación de la aféresis: su elevado coste. Recientemente se ha publicado un estudio de coste-efectividad por Panés y cols. (40) que demuestra que el tratamiento es coste efectivo frente al tratamiento convencional con esteroides e inmunomoduladores en pacientes con CU corticodependiente y corticorresistente; sin embargo, este estudio de fármaco-economía se ha realizado considerando una pauta terapéutica de 5 sesiones de GMCAP.

Por lo tanto, sería interesante identificar parámetros predictores de buena respuesta al tratamiento con el fin de poder seleccionar mejor a los pacientes. En la actualidad los pacientes que mejor responden a la granulocitoaféresis son aquellos con el primer brote de $\mathrm{CU}$, sin esteroides previos, corticodependientes, con una enfermedad de corta evolución y cifras elevadas de proteína $\mathrm{C}$ reactiva al inicio del tratamiento $(7,41)$.

En conclusión, la granulocitoaféresis es un tratamiento inmunomodulador bien tolerado, que posee escasos efectos secundarios y con buenos resultados en el tratamiento de la CU corticodependiente, corticorresistente, en pacientes "nä̈ve", en la población pediátrica e incluso podría contemplarse su uso en el embarazo. Sus resultados son menos claros en la EC. Aún existen puntos por dilucidar como el número de sesiones, la duración de las mismas y un posible tratamiento de mantenimiento. Es necesario realizar estudios de fármaco-economía y determinar su lugar en el algoritmo del tratamiento de la EII. Probablemente en un futuro se desarrollarán estrategias de tratamientos combinados de GMCAP con inmunosupresores y/o terapias biológicas.

M. P. Martínez Montiel

\section{Servicio de Aparato Digestivo. Hospital 12 de Octubre. Madrid}

\section{Bibliografía}

1. Solem CA, Loftus EV Jr. Management of refractory inflammatory bowel disease. Gastroenterol Clin North Am 2004; 33: 319-34.

2. Faubion WA, Loftus EV, Harmsen WS, et al. The natural history of corticosteroid therapy for inflammatory bowel disease: a population-based study. Gastroenterol 2001; 121: 255-60.

3. Hiraishi K, Takeda Y, Saniabadi A, et al. Studies on the mechanisms of leukocyte adhesion to cellulose acetate beads: An in vitro model to asses the efficacy and monocyte adsortive apheresis. Ther Apher Dial 2003; 7: 334-40.

4. Ohara M, Saniabadi AR, Kokuma S, et al. Granulocytapheresis in the treatment of patients with rheumatoid arthritis. Artif Organs 1997; 2: 989-94.

5. Sawada K, Ohnishi K, Kosaka T, et al. Leukocytapheresis with leukocyte removal filter as new therapy for ulcerative colitis. Ther Apher 1997; 1: 207-11.

6. Tibble JA, Sigthorsson G, Bridger DS, et al. Surrogate markers of intestinal inflammation are predictive of relapse in patients with inflammatory bowel disease. Gastroenterol 2000; 119: 15-22.

7. Shimoyama T, Sawada K, Hiwatasi N, et al. Safety and efficacy of granulocyte and monocyte adsorption apheresis in patients with active ulcerative colitis: A multicenter study. J Clin Apher 2001; 16: 1-9.

8. Hanai H. Positions of selective leukocytapheresis in the medical therapy of ulcerative colitis. World J Gastroenterol 2006; 12 (47): 7568-77.

9. Suzuki Y, Yoshimura N, Saniabadi AR, et al. Selective granulocyte and monocyte adsortive apheresis as a firstline treatment fot steroid naive patients with active ulcerative colitis: A prospective uncontrolled study. Dig Dis Sci 2004; 49: 565-71. 
10. Hanai H, Watanabe F, Saniabadi AR, et al. Therapeutic efficacy of granulocyte adsorption apheresis in severe active ulcerative colitis. Dig Dis Sci 2002; 47: 2349-53.

11. Saniabadi AR, Hanai H, Bjarnason I, et al. Adacolum, an adsortive carrier based granulocyte and monocyte apheresis device for the treatment of infllammatory and refractory diseases associated with leukocytes. Ther Apher Dial 2003; 7: 48-59.

12. Hanai H, Iida T, Yamada M, et al. Effects of adacolum selective leukocytapheresis on plasma cytokines during active disease in patients with active ulcerative colitis. World J Gastroenterol 2006; 12: 3393-9.

13. Saniabadi AR, Hanai H, Bjarnason I, et al. Adacolum for selective leukocytapheresis a non-pharmacological treatment for patients with disorders of the immune system: An adjunct or an alternative to drug therapy? J Clin Apher 2005; 20: 171-84.

14. Muratov V, Lundahl J, Ufgren AK, et al. Down-regulation of interferon- $\gamma$ parallels clinical response to selective leukocyte apheresis in patients with inflammatory bowel deisease: A 12-month follow-up study. Int J Colorectal Dis 2005. DOI 10.1007/s00384-005-0069-2.

15. Powrie F, Read S, Mottet C, et al. Control of immune pathology by regulatory T cells. Novartis Found Symp 2003; 252: 92-8; discusión 98-105: 106-14.

16. Kanai K, Watanabe M. Clinical application of human CD4+CD25+ regulatory T cells for the treatment of inflammatory bowel diseases. Expert Opin Biol Ther 2005; 5: 451-62.

17. Huibregtse IL, van Lent AU, van Deventer SJH. Inmunopathogenesis of IBD: insufficient supressor function in the gut? Gut 2007; 56: 584-92.

18. Kanai T, Hibi T, Watanabe M. The logics of leukocytapheresis as a natural biological therapy for inflammatory bowel disease. Expert Opin Biol Ther 2006; 6 (5): 453-66.

19. Hanai H, Watanabe F, Takeuchi K, et al. Leukocyte adsortive apheresis for the treatment of active ulcerative colitis: a prospective, uncontrolled, pilot study. Clin Gastroenterol Hepatol 2003; 1: 28-35.

20. Durai D, Hawthorne AB. Review article: How and when to use ciclosporin in ulcerative colitis. Aliment Pharmacol Ther 2005; 22 (10): 907-16.

21. Kanke K, Nakano M, Hirasi H, et al. Evaluation of granulocyte/monocyte apheresis therapy for active ulcerative colitis. Dig Liv Dis 2004; 36: 512-8.

22. Naganuma M, Funakoshi S, Sakuraba A, et al. Granulocytapheresis is useful as an alternative therapy in patients with steroid-refractory or depent ulcerative colitis. Inflamm. Bowel Dis 2004; 10: 251-7.

23. Yamamoto T, Umegaes S, Kitagawa T, et al. Granulocyte and monocyte adsortive apheresis of active distal ulcerative colitis: A propective pilot study. Aliment Pharmacol Ther 2004; 20: 783-92.

24. Premchand P, Takeuchi K, Bjarnason I. Granulocyte, macrophage, monocyte apheresis for refractory ulcerative proctitis. Eur J Gastroenterol Hepatol 2004; 16: 943-5.

25. Hanai H, Watanabe F, Yamada M, et al. Adsortive granulocyte and monocyte apheresis versus prednisolone in patients with corticosteroid-dependent moderaly severa ulcerative colitis. Digestion 2004; 70: 36-44.

26. Bresci G, Parisi G, Mazzoni A, et al. Treatment of patients with acute ulcerative colitis: convencional corticosteroid therapy (MP) versus granulocytapheresis (GMA): A pilot study. Dig Liver Dis 2007; 39: 430-4.

27. Caprilli R, D’Ovidio V. Leukocytoapheresis as promising therapy for inflammatory bowel disease. Digest Liver Dis Rev. 2007 (en prensa).

28. Matsui T, Nishimura T, Matake H, et al. Granulocytapheresis for Crohn's disease: a report on seven refractory patients. Am J Gastroenterol 2003; 98: 511-2.

29. Fukuda Y, Matsui T, Suzuki Y, et al. Adsortive granulocyte and monocyte apheresis for refractory Crohn's disease: An open multicenter prospective study. J Gastroenterol 2004; 39: 1158-64.

30. Maiden L, Baur R, Takeuchi K, et al. Adacolum apheresis reduces relapse rates in patient with IBD at significant risk of clinical relapse rates in patients with IBD at significant risk of clinical relapse (abstract). Gastroenterol 2005; 128: A581.

31. Domenech E, Hinojosa J, Esteve-Comas M, et al. Spanish Group for the Study of Crohn's Disease and Ulcerative Colitis (GETECCU). Granulocyteapheresis in steroid-dependent imflammatory bowel disease: A prospective, open, pilot study. Aliment Pharmacol Ther 2004; 20: 1347-52.

32. Fernández-Pérez FJ, Rodríguez F, De Sola C, et al. Granulocitaféresis en la enfermedad inflamatoria intestinal. Eficacia a 32 semanas con protocolo de inducción y sesiones de mantenimiento. Rev Esp Enferm Dig 2007; 99 : 628-35.

33. Cabriada JL, Domenech E, Gomollon F, et al. GETECCU. Documento de consenso en el uso de la granulocitoaféresis en pacientes con enfermedad inflamatoria intestinal. Gastroenterol Hepatol 2006; 29: 85-92.

34. Yamamoto T, Umegae S, Matsumoto K. Safety and clinical efficacy of granulocyte and monocyte adsortive apheresis therapy for ulcerative colitis. World J Gatroenterol 2006; 12: 520-5.

35. Tomomasa T, Kobayashi A, Kanedo H, et al. Granulocyte adsorptive apheresis for pediatric patients with ulcerative colitis. Dig Dis Sci 2003; 48: 750-4.

36. Ikeda H, Ishimaru Y, Takayasu H, et al. Efficacy of granulocyte apheresis in pediatric patients with ulcerative colitis: A pilot study. Jorunal of Pediatric Gastroenterology and Nutrition 2006; 43: 592-6.

37. Okada H, Makidono C, Takenaka R, et al. Therapeutic efficacy of leukocytapheresis in a pregnant woman with severe active ulcerative colitis. Digestion 2006; 74: 15-8.

38. Sakuraba A, Naganuma M, Hibi T. Intensive therapy of granulocyte and adsorption apheresis induces rapid remission in patients with ulcerative colitis. Gastroenterol 2003; 124 (Supl. 1): A522.

39. Ricart E, Esteved M, Andreu M, et al. Evaluation of 5 versus 10 granulocyteapheresis sessions in steroi-dependent ulcerative colitis: A pilot, propective, multicenter, randomized study. World J Gastroenterol 2007; 13: 2193-7.

40. Panés J, Guilera M, Ginard D, et al. Treatment cost of ulcerative colitis. Is apheresis with Adacolun cost-effective? Dig Liver Dis 2007; 39; 617-25.

41. D'Ovidio V, Arati A, Viscido A, et al. Long term follow-up in ulcerative colitis patients undergoing granulocyte-monocyte apheresis for steroid dependency/resistancy. Gut 2006; 55 (Supl. 5): A-123. 